\title{
Terahertz oscillators using electron devices - an approach with Resonant tunneling diodes
}

\author{
Masahiro Asada ${ }^{\text {a) }}$ and Safumi Suzuki \\ Interdisciplinary Graduate School of Science and Engineering, Tokyo Institute of \\ Technology \\ 2-12-1-S9-3 Ookayama, Meguro-ku, Tokyo 152-8552, Japan \\ a)asada@pe.titech.ac.jp
}

\begin{abstract}
Resonant tunneling diodes (RTDs) have the potential for compact and coherent terahertz $(\mathrm{THz})$ sources operating at room temperature. In this paper, recent results of $\mathrm{THz}$ oscillators with RTDs are described. A fundamental oscillation frequency up to $831 \mathrm{GHz}$ was achieved with RTD having high available current density and low capacitance. By the structure reducing the transit time, the frequency further increased to $1.04 \mathrm{THz}$. This is the first achievement of a fundamental oscillation above $1 \mathrm{THz}$ in room-temperature electronic single oscillators. The output power of $400 \mu \mathrm{W}$ at $550 \mathrm{GHz}$ was obtained in a single oscillator by the offset-fed slot antenna. Coherent power combining with multi-element array was observed. The spectral linewidth, frequency change with bias voltage, and direct modulation were also described.
\end{abstract}

Keywords: terahertz oscillator, electron devices, resonant tunneling diode

Classification: Electron devices, circuits, and systems

\section{References}

[1] M. Tonouchi, "Cutting-edge terahertz technology," Nat. Photonics, vol. 1, pp. 97-105, 2007.

[2] S. Komiyama, "Far-Infrared Emission from Population-Inverted HotCarrier System in p-Ge," Phys. Rev. Lett., vol. 48, pp. 271-274, 1982.

[3] R. Köhler, A. Tredicucci, F. Beltram, H. E. Beere, E. H. Linfeld, A. G. Davies, D. A. Ritchie, R. C. Iotti, and F. Rossi, "Terahertz semiconductor heterostructure laser," Nature, vol. 417, pp. 156-159, 2002.

[4] B. S. Williams, "Terahertz quantum-cascade lasers," Nat. Photonics, vol. 1, pp. 517-525, 2007.

[5] J. Nishizawa, P. Plotka, T. Kurabayashi, and H. Makabe, "Development of TUNNETT Diode as Terahertz Device and Its Applications," Annual Device Research Conf., V. A-3, Pennsylvania, June 2006.

[6] H. Eisele and R. Kamoua, "Submillimeter-Wave InP Gunn Devices," IEEE Trans. Microw. Theory Tech., vol. 52, no. 10, pp. 2371-2378, Oct. 2004. 
[7] H. Eisele, "480 GHz oscillator with an InP Gunn device," Electron. Lett., vol. 46 , no. 6 , pp. 422-423, 2010.

[8] E. R. Brown, J. R. Sönderström, C. D. Parker, L. J. Mahoney, K. M. Molvar, and T. C. McGill, "Oscillations up to $712 \mathrm{GHz}$ in InAs/AISb resonant-tunneling diodes," Appl. Phys. Lett., vol. 58, pp. 2291-2293, 1991.

[9] M. Reddy, S. C. Martin, A. C. Molnar, R. E. Muller, R. P. Smith, P. H. Siegel, M. J. Mondry, M. J. W. Rodwell, H. Kroemer, and S. J. Allen, "Monolithic Schottky-Collector Resonant Tunnel Diode Oscillator Arrays to $650 \mathrm{GHz}$," IEEE Electron Device Lett., vol. 18, pp. 218-221, 1997.

[10] M. Asada, S. Suzuki, and N. Kishimoto, "Resonant Tunneling Diodes for Sub-Terahertz and Terahertz Oscillators," Jpn. J. Appl. Phys., vol. 47, no. 6, pp. 4375-4384, 2008.

[11] N. Orihashi, S. Suzuki, and M. Asada, "One THz harmonic oscillation of resonant tunneling diodes," Appl. Phys. Lett., vol. 87, 233501, 2005.

[12] S. Suzuki, A. Teranishi, K. Hinata, M. Asada, H. Sugiyama, and H. Yokoyama, "Fundamental Oscillation of up to $831 \mathrm{GHz}$ in GaInAs/AlAs Resonant Tunneling Diode," Appl. Phys. Exp., vol. 2, 0545012009.

[13] S. Suzuki, M. Asada, A. Teranishi, H. Sugiyama, and H. Yokoyama, "Fundamental oscillation of resonant tunneling diodes above $1 \mathrm{THz}$ at room temperature," Appl. Phys. Lett., vol. 97, 242102, 2010.

[14] R. Lai, W. R. Deal, X. B. Mei, W. Yoshida, J. Lee, L. Dang, J. Wang, Y. M. Kim, P. H. Liu, V. Radisic, M. Lange, T. Gaier, L. Samoska, and A. Fung, "Fabrication of InP HEMT Devices with Extremely High Fmax," Int. Conf. IPRM08, MoA3.2, Versailles, May 2008.

[15] D.-H. Kim and J. A. del Alamo, "30-nm InAs Pseudomorphic HEMTs on an InP Substrate With a Current-Gain Cutoff Frequency of $628 \mathrm{GHz}$," IEEE Electron Device Lett., vol. 29, no. 8, pp. 830-833, 2008.

[16] V. A. Leuther, S. Koch, A. Tessmann, I. Kallfass, T. Merkle, H. Massler, R. Loesch, M. Schlechtweg, S. Saito, and O. Ambacher, "20 nm Metamophic HEMT with $660 \mathrm{GHz} f \mathrm{ft}$, Int. Conf. Indium Phosphide and Related Materials, Tu-4.2.2, Berlin, Germany, May 2011.

[17] V. M. Urteaga, M. Seo, J. Hacker, Z. Griffith, A. Young, R. Pierson, P. Rowell, A. Skalare, V. Jain, E. Lobisser, and M. J. W. Rodwell, "InP HBTs for THz Frequency Integrated Circuits," Int. Conf. Indium Phosphide and Related Materials, Mo-1.2.1, Berlin, Germany, May 2011.

[18] J. Hacker, M. Urteaga, D. Mensa, R. Pierson, M. Jones, Z. Griffith, and M. Rodwell, "250 nm InP DHBT Monolithic Amplifiers with 4.8 dB Gain at $324 \mathrm{GHz}, "$ IEEE MTT-S Digest, pp. 403-406, 2008.

[19] V. Radisic, D. Sawdai, D. Scott, W. R. Deal, L. D. Danny Li, J. Chen, A. Fung, L. Samoska, T. Gaier, and R. Lai, "Demonstration of a 311GHz Fundamental Oscillator Using InP HBT Technology," IEEE Trans. Microw. Theory Tech., vol. 55, no. 11, pp. 2329-2335, Nov. 2007.

[20] V. Radisc, L. Samoska, W. R. Deal, X. B. Mei, W. Yoshida, P. H. Liu, J. Uyeda, A. Fung, T. Gaier, and R. Lai, "A 330-GHz MMIC Oscillator Module," IEEE MTT-S, Dig., p. 395, 2008.

[21] W. R. Deal, X. B. Mei, V. Radisic, K. Leong, S. Sarkozy, B. Gorospe, J. Lee, P. H. Liu, W. Yoshida, J. Zhou, M. Lange, J. Uyeda, and R. Lai, "Demonstration of a $0.48 \mathrm{THz}$ Amplifier Module Using InP HEMT Transistors," IEEE Microw. Wireless Compon. Lett., vol. 20, no. 5, pp. 289291, May 2010.

[22] E. Seok, C. Cao, D. Shim, D. J. Arenas, D. B. Tanner, C.-M. Hung, and K. K. O, "A $410 \mathrm{GHz}$ CMOS Push-Push Oscillator with an On-Chip 
Patch Antenna," ISSCC, 26.1, San Francisco, Feb. 2008.

[23] B. Razavi, "A 300-GHz Fundamental Oscillator in 65-nm CMOS Technology," Symp. VLSI Circuits Dig., pp. 113-114, 2010.

[24] Q. J. Gu, Z. Xu, H.-Y. Jian, X. Xu, M.-C. F. Chang, W. Liu, and H. Fetterman, "Generating Terahertz Signals in 65nm CMOS with Negative-Resistance Resonator Boosting and Selective Harmonic Suppression," Symp. VLSI Circuits Dig., pp. 109-110, 2010.

[25] L. Esaki and R. Tsu, "Superlattice and Negative Differential Conductivity in Semiconductors," IBM J. Res. Dev., vol. 14, pp. 61-65, 1970.

[26] N. Sekine and K. Hirakawa, "Dispersive Terahertz Gain of a Nonclassical Oscillator: Bloch Oscillation in Semiconductor Superlattices," Phys. Rev. Lett., vol. 94, 057408, 2005.

[27] T. Otsuji, Y. M. Meziani, M. Hanabe, T. Ishibashi, T. Uno, and E. Sano, "Grating-bicoupled plasmon-resonant terahertz emitter fabricated with GaAs-based heterostructure material systems," Appl. Phys. Lett., vol. 89, 263502, 2006 .

[28] J. Takeuchi, Y. Iwahashi, and M. Asada, "Fabrication and Millimeterwave Characterization of Semiconductor Klystron Device Using TwoDimensional Electron Gas," Topical Workshop on Heterostructure Materials, ThB-3, Kisarazu, Japan, Aug. 2007.

[29] R. Tsu and L. Esaki, "Tunneling in a finite superlattice," Appl. Phys. Lett., vol. 22, no. 11, pp. 562-564, 1973.

[30] L. L. Chang, L. Esaki, and R. Tsu, "Resonant Tunneling in Semiconductor Double Barriers," Appl. Phys. Lett., vol. 24, no. 12, pp. 593-595, 1974.

[31] M. Tsuchiya, H. Sakaki, and J. Yoshino, "Room Temperature Observation of Differential Negative Resistance in an AlAs/GaAs/AlAs Resonant Tunneling Diode," Jpn. J. Appl. Phys., vol. 24, no. 6, pp. L466-L468, 1985.

[32] T. C. L. G. Sollner, P. E. Tannenwald, D. D. Peck, and W. D. Goodhue, "Quantum well oscillators," Appl. Phys. Lett., vol. 45, pp. 1319-1321, 1984.

[33] H. C. Liu and T. C. L. G. Sollner, in "High-Speed Heterostructure Device," ed. R. A. Kiehl and T. C. L. G. Sollner, ch.6 and references therein, Academic, San Diego, 1994.

[34] K. Hinata, M. Shiraishi, S. Suzuki, M. Asada, H. Sugiyama, and H. Yokoyama, "Sub-Terahertz Resonant Tunneling Diode Oscillators with High Output Power $(\sim 200 \mu \mathrm{W})$ Using Offset-Fed Slot Antenna and High Current Density," Appl. Phys. Express, vol. 3, 014001, 2010.

[35] M. Shiraishi, H. Shibayama, K. Ishigaki, S. Suzuki, M. Asada, H. Sugiyama, and H. Yokoyama, "High Output Power $(\sim 400 \mu \mathrm{W})$ Oscillators at around $550 \mathrm{GHz}$ Using Resonant Tunneling Diodes with Graded Emitters and Thin Barriers," Appl. Phys. Express, vol. 4, 064101, 2011.

[36] N. Orihashi, S. Hattori, and M. Asada, "Millimeter and Submillimeter Oscillators Using Resonant Tunneling Diodes with Stacked-Layer Slot Antennas," Jpn. J. Appl. Phys., vol. 43, no. 10A, pp. L1309-L1311, 2004.

[37] K. Urayama, S. Aoki, S. Suzuki, M. Asada, H. Sugiyama1, and H. Yokoyama, "Sub-Terahertz Resonant Tunneling Diode Oscillators Integrated with Tapered Slot Antennas for Horizontal Radiation," Appl. Phys. Express, vol. 2, 044501, 2009.

[38] R. Sekiguchi, Y. Koyama, and T. Ouchi, "Subterahertz oscillations from triple-barrier resonant tunneling diodes with integrated patch antennas," Appl. Phys. Lett., vol. 96, 062115, 2010.

[39] N. Kishimoto, S. Suzuki, A. Teranishi, and M. Asada, "Frequency In- 
crease of Resonant Tunneling Diode Oscillators in Sub- $\mathrm{THz}$ and $\mathrm{THz}$ Range Using Thick Spacer Layers," Appl. Phys. Express, vol. 1, 042003, 2008.

[40] S. Suzuki, K. Sawada, A. Teranishi, M. Asada, H. Sugiyama, and H. Yokoyama, "Fundamental oscillations at $\sim 900 \mathrm{GHz}$ with low bias voltages in RTDs with spike-doped structures," Electron. Lett., vol. 46, no. 14, pp. 1006-1007, July 2010.

[41] A. Teranishi, K. Shizuno, S. Suzuki, M. Asada, H. Sugiyama, and H. Yokoyama, "Fundamental Oscillation up to $1.08 \mathrm{THz}$ in Resonant Tunneling Diodes with High Indium Composition Transit Layers," Int. Conf. IPRM2011, P-09, Berlin, May 2011.

[42] C. S. Kim and A. Brändli, "High-Frequency High-Power Operation of Tunnel Diodes," IRE Trans. Circuit Theory, vol. 8, pp. 416-425, 1961.

[43] S. Suzuki and M. Asada, "Proposal of Resonant Tunneling Diode Oscillators with Offset-Fed Slot Antennasin Terahertz and Sub-Terahertz Range," Jpn. J. Appl. Phys., vol. 46, no. 1, pp. 119-121, 2007.

[44] S. Suzuki, N. Kishimoto, M. Asada, N. Sekine, and I. Hosako, "Experimental and Theoretical Investigation of the Dependence of Oscillation Characteristics on Structure of Integrated Slot Antennas in Sub-terahertz and Terahertz Oscillating Resonant Tunneling Diodes," Jpn. J. Appl. Phys., vol. 47, no. 1 pp. 64-67, 2008.

[45] S. Suzuki, N. Orihashi, and M. Asada, "Mutual Injection Locking between Sub-THz Oscillating Resonant Tunneling Diodes," Jpn. J. Appl. Phys., vol. 44, no. 48, pp. L1439-L1441, 2005.

[46] S. Suzuki and M. Asada, "Coherent Power Combination in Highly Integrated Resonant Tunneling Diode Oscillatorswith Slot Antennas," Jpn. J. Appl. Phys., vol. 46, no. 46, pp. L1108-L1110, 2007.

[47] K. D. Stephan, S.-C. Wang, E. R. Brown, K. M. Molvar, A. R. Calawa, and M. J. Manfra, "5mW Parallel-Connected Renent-Tunneling-Diode Oscillator," Electron. Lett., vol. 28, no. 15, pp. 1411-1412, July 1992.

[48] D. P. Steenson, J. M. Chamberlain, R. E. Miles, R. D. Pollard, and M. Henini, "Demonstration of Power Combining at W-band from GaAs/AlAs Resonant Tunnelling Diodes," Int. Conf. Superlattices, Microstructure and Microdevices (ICSMM-7), Banff, Canada, pp. 98-99, Aug. 1994.

[49] M. P. DeLisio, J. F. Davis, S-J. Li, D. B. Rutledge, and J. J. Rosenberg, "A 16-Element Tunnel Diode Grid Oscillator," IEEE AP-S, Int. Symp., p. 1284, 1995.

[50] T. Fujii, H. Mazaki, F. Takei, J. Bae, M. Narihiro, T. Noda, H. Sakaki, and K. Mizuno, "Coherent Power Combining of Millimeter Wave Resonant Tunneling Diodes in a Quasi-Optical Resonator," IEEE MTT-S, Int. Microwave Symp., WE3F-28, p. 919, 1996.

[51] H. I. Cantu and W. S. Trunscott, "Injection-locking and power combining with double barrier resonant tunnelling diodes," Electron. Lett., vol. 37, no. 20, pp. 1264-1265, Sept. 2001.

[52] M. Asada and S. Suzuki, "Theoretical analysis of coupled oscillator array using resonant tunneling diodes in subterahertz and terahertz range," $J$. Appl. Phys., vol. 103, 124514, 2008.

[53] S. Suzuki, K. Urayama, and M. Asada, "Coherent Power Combination in Multi-Element Sub-Terahertz Resonant Tunneling Diode Oscillators Coupled with Metal-Insulator-Metal Stub Structure," Appl. Phys. Express, vol. 1, 093001, 2008.

[54] K. Karashima, R. Yokoyama, M. Shiraishi, S. Suzuki, S. Aoki, and M. Asada, "Measurement of Oscillation Frequency and Spectral Linewidth 
of Sub-Terahertz InP-Based Resonant Tunneling Diode Oscillators Using Ni-InP Schottky Barrier Diode," Jpn. J. Appl. Phys., vol. 49, 020208, 2010.

[55] K. Karashima, M. Shiraishi, K. Hinata, S. Suzuki, and M. Asada, "Heterodyne Detection of Output of Sub-THz RTD Oscillator Using InP-SBD Detector and RTD Local Oscillator," Int. Conf. IRMMW-THz, Th-P76, Rome, Sept. 2010.

[56] M. Asada, "Theoretical analysis of spectral linewidth of terahertz oscillators using resonant tunneling diodes and their coupled arrays," J. Appl. Phys., vol. 108, 034504, 2010.

[57] N. Shimizu, T. Waho, and T. Ishibashi, "Capacitance anomaly in the negative differential resistance region of resonant tunneling diodes," Jpn. J. Appl. Phys., vol. 36, no. 3B, pp. L330-L333, 1997.

[58] T. Wei, S. Stapleton, and E. Berolo, "Equivalent circuit and capacitance of double barrier resonant tunneling diode," J. Appl. Phys., vol. 73, no. 2, pp. 829-834, Jan. 1993.

[59] R. Lake and J. Yang, "A Physics Based Model for the RTD Quantum Capacitance," IEEE Trans. Electron Devices, vol. 50, no. 3, pp. 785-789, 2003.

[60] N. Orihashi, S. Hattori, S. Suzuki, and M. Asada, "Voltage-controlled sub-terahertz oscillation of resonant tunnelling diode integrated with slot antenna," Electron. Lett., vol. 41, no. 15, pp. 872-873, July 2005.

[61] M. Asada, N. Orihashi, and S. Suzuki, "Experiment and Theoretical Analysis of Voltage-Controlled Sub-THz Oscillation of Resonant Tunneling Diodes," IEICE Trans. Electron., vol. E89-C, pp. 968-975, 2006.

[62] T. Kleine-Ostmann and T. Nagatsuma, "A Review on Terahertz Communications Research," J. Infrared Millimeter and Terahertz Waves, vol. 32, pp. 143-171, 2011.

[63] K. Ishigaki, K. Karashima, M. Shiraishi, H. Shibayama, S. Suzuki, and M. Asada, "Direct Modulation of THz-Oscillating Resonant Tunneling Diodes," to be presented at Int. Conf. IRMMW-THz, Huston, Oct. 2011.

\section{Introduction}

The terahertz $(\mathrm{THz})$ frequency range located approximately between 0.1$1 \mathrm{THz}$ has been receiving considerable attention recently because of its many applications, such as ultrahigh-speed wireless communications, spectroscopy, and imaging [1]. For these applications, compact and coherent solid-state sources are important key components. Because the $\mathrm{THz}$ range is located between lightwaves and millimeter waves, both optical and electronic devices are being investigated for $\mathrm{THz}$ sources. For semiconductor single oscillators, p-type Ge lasers [2] and THz quantum cascade lasers (QCLs) [3, 4] are studied from the optical device side.

From the electron device side, the development of two-terminal devices such as impact ionization avalanche transit-time (IMPATT) diodes, tunneling transit-time (TUNNETT) diodes, Gunn diodes, and resonant tunneling diodes (RTDs) is being pursued [5, 6, 7, 8, 9, 10, 11, 12, 13]. Heterostructure bipolar transistos (HBTs), high electron mobility transistors (HEMTs), and Si C-MOS transistors are also studied intensively [14, 15, 16, 17, 18, 19, 20, 
$21,22,23,24]$. The maximum oscillation frequency, i. e., the frequency at which the power gain $=0 \mathrm{~dB}$, of the InP-based HEMT exceeded $1 \mathrm{THz}$ [14]. Sub- $\mathrm{THz}$ oscillation and amplification were reported in MMICs with InPbased HEMTs and HBTs [18, 19, 20, 21]. Oscillators with Si C-MOS were also studied [22, 23, 24]. Devices based on Bloch oscillation, plasma effect, and velocity modulation are also being studied [25, 26, 27, 28]. Fig. 1 shows output power versus oscillation frequency for several semiconductor single oscillators at this stage.

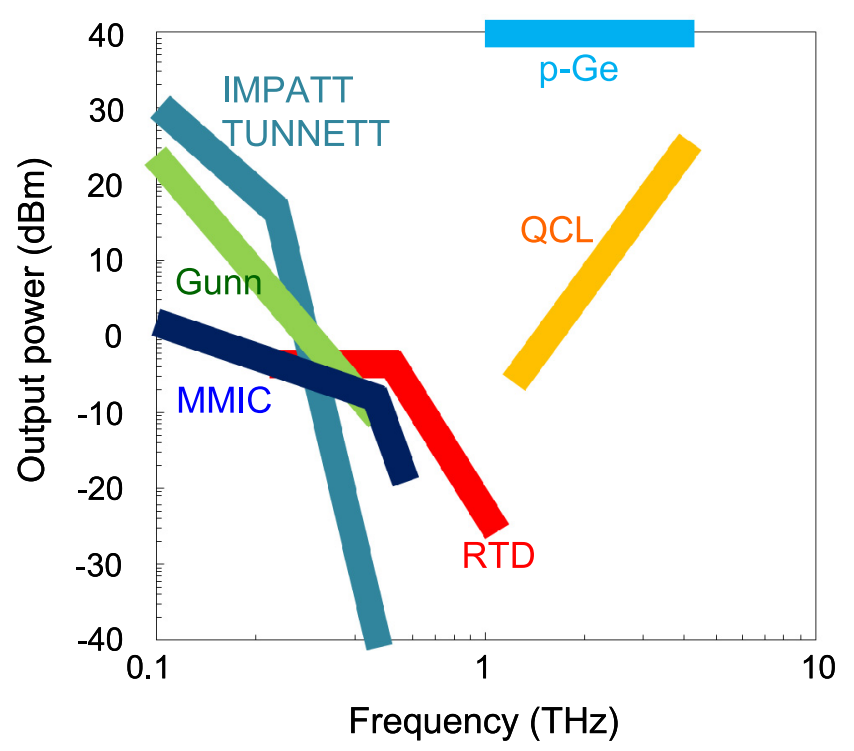

Fig. 1. Output power as a function of frequency for several semiconductor $\mathrm{THz}$ sources at this stage. Recent results in RTD oscillators described in this paper are included.

RTDs have the highest oscillation frequency among the above electron devices. Room-temperature operation, which has not yet been achieved in a single optical device at present, is also an attractive feature. Research of RTDs began with the theoretical prediction by Tsu and Esaki in 1973 [29], and their behavior of negative differential resistance was experimentally demonstrated at liquid nitrogen temperature in 1974 [30] and at room temperature in 1985 [31]. Oscillation in the microwave range was demonstrated at a low temperature in 1984 [32]. The oscillation frequency was then updated many times to several hundred $\mathrm{GHz}$ [33], and a room-temperature fundamental oscillation at $712 \mathrm{GHz}$ was reported in 1991 [8]. Although the increase in oscillation frequency stopped after this achievement until recently, an oscillation at $831 \mathrm{GHz}$ was reported in 2009 [12], and then, $1.04 \mathrm{THz}$ was achieved very recently [13]. The output power has also increased by improvement of the structures of RTD and resonator [34, 35].

In this paper, we describe recent results of $\mathrm{THz}$ oscillators using RTDs. The operation principle and fabricated structures of RTDs, and oscillation characteristics are described in Section 2. Structures for high frequency oscil- 
lation and high output power are discussed in Sections 3 and 4, respectively. Spectral linewidth, frequency change with bias voltage, and direct modulation are shown in Section 5. Conclusion is given in Section 6 .

\section{Device structure and oscillation characteristics}

\subsection{Oscillation principle and device structure}

The RTD is normally composed of two heterobarriers and a quantum well, as shown in Fig. 2 (a). In the current-voltage $(I-V)$ characteristics shown schematically in Fig. 2 (b), a current peak exists at which the resonance level in the quantum well is close to the conduction band edge of the emitter. With increasing voltage above the current peak, the $I-V$ curve exhibits a negative differential conductance (NDC) region. The oscillator utilizes this region.

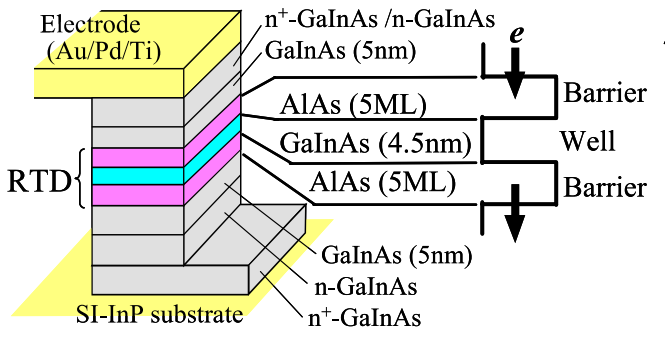

(a)

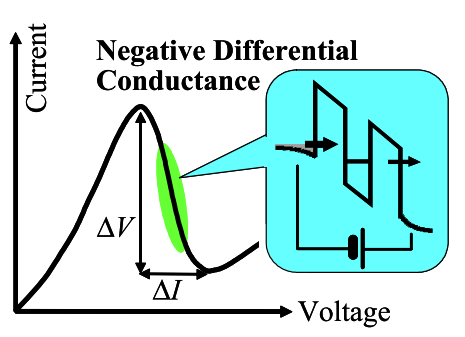

(b)

Fig. 2. Fundamental structure and characteristics of RTD. (a) An example of layer structure of doublebarrier RTD and (b) current-voltage characteristics.

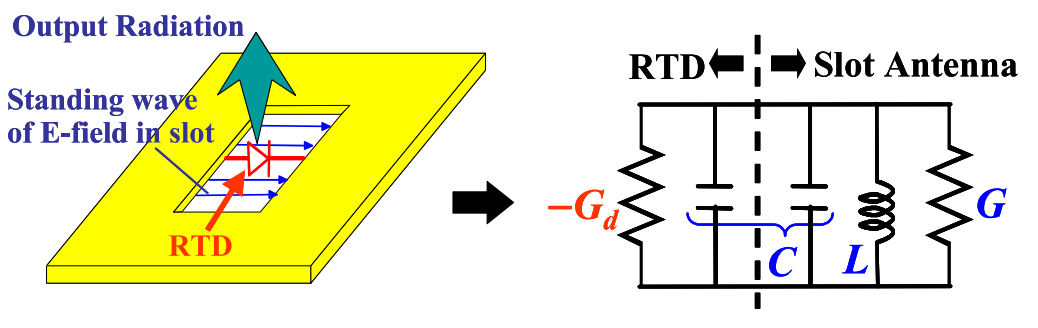

(a)

(b)

Fig. 3. Principle of RTD oscillator. (a) Slot resonator and RTD and (b) its equivalent circuit.

Figure 3 (a) shows the fundamental structure of the RTD oscillator integrated with a planar slot resonator. The RTD is located at the center of a slot in a metal film. This slot forms a standing wave of the electromagnetic field as a resonator, and also acts as an antenna by radiating output power at the same time. The equivalent circuit for this structure is illustrated in Fig. 3 (b). Parasitic elements are neglected in the figure except the capacitance of the 
RTD. Oscillation takes place if the absolute value of NDC exceeds the radiation loss of the slot antenna. The oscillation frequency is determined by the parallel resonance of the $L C$ circuit in Fig. 3 (b) corresponding to formation of the standing wave in Fig. 3 (b).

The actual structure of the fabricated device is shown in Fig. 4 [10, 11, 12, 13]. The electrodes of the RTD are connected to the left and right electrodes of the antenna. At both edges of the antenna, the electrodes are overlapped with a $\mathrm{SiO}_{2}$ layer between them. Using this structure, reflectors of highfrequency electromagnetic waves are formed, and the separation of DC bias is achieved at the same time. The length of the slot antenna is 10-50 $\mu \mathrm{m}$. A parallel resistance with a bismuth film is connected outside the antenna electrodes to suppress parasitic oscillation of $2-3 \mathrm{GHz}$ due to the resonance formed by external circuits including bias supplying lines. The area of the RTD mesa is around $0.5-1 \mu \mathrm{m}^{2}$. The output power was measured at the bottom side of the substrate through a hemispherical Si lens (see Fig. 12), because most of the output power is radiated into the substrate due to its large dielectric constant. Lens-free oscillators were also reported [36, 37, 38].
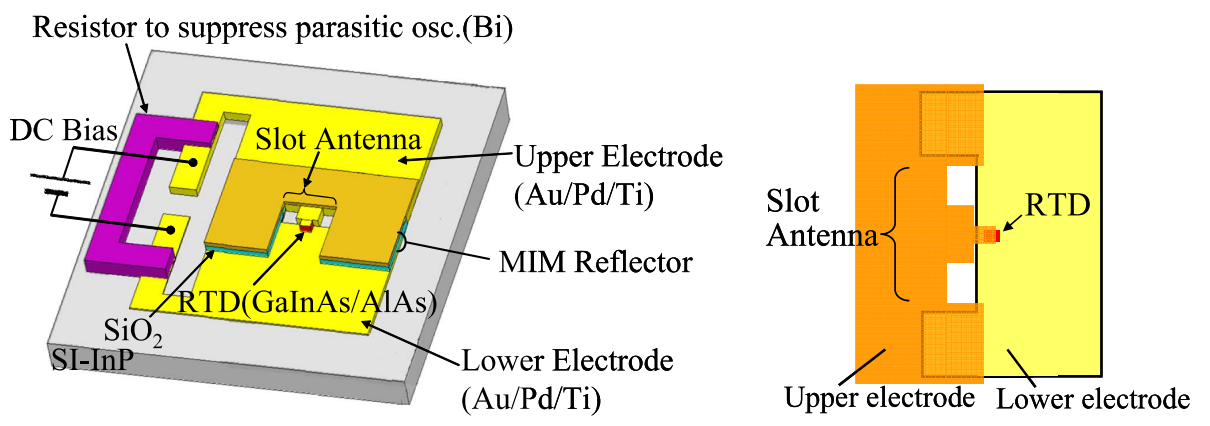

Fig. 4. Fabricated structure of RTD oscillator with slot antenna.

\subsection{Oscillation characteristics}

To obtain high frequency oscillation, the capacitance of RTD shown in Fig. 3 (b) has to be reduced. For this purpose, we introduced a thick spacer layer in the collector region adjacent to the resonant tunneling layers, as shown in Fig. 5 (a) [12, 39]. The RTD mesa area must also be reduced. However, it becomes difficult to obtain high output power and to satisfy the oscillation condition because of reduction in the available current $(\Delta I$ in Fig. 2(b)). Therefore, high available current density is required at the same time. In the structure in Fig. 5 (a), this requirement was met with high doping into the emitter region.

The measured $I-V$ curve of the structure in Fig. 5 (a) is shown in Fig. 5 (b), which is asymmetry with respect to the polarity because of the difference in thicknesses of the collector and emitter layers. Fig. 5 (c) shows the observed spectra of fundamental oscillations at room temperature. A liquid He-cooled Si composite bolometer was used as a detector and the os- 


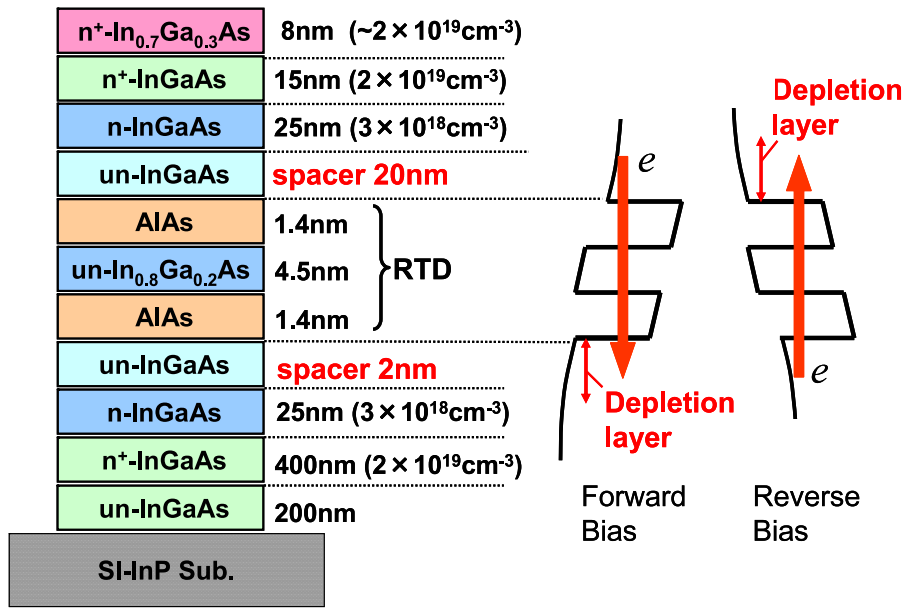

(a)

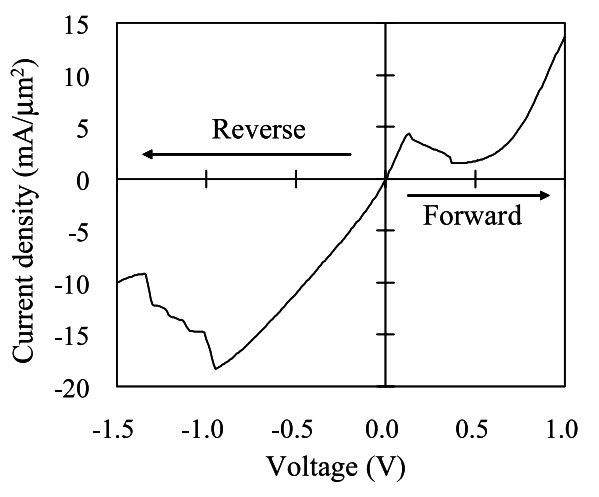

(b)

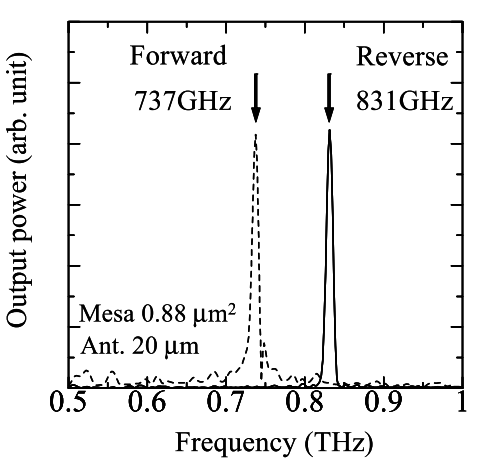

(c)

Fig. 5. RTD with thick spacer layer for high frequency oscillation. (a) Layer structure, (b) measured $I-$ $V$ characteristics, and (c) oscillation spectra.

cillation spectra were measured by a Fourier transform infrared spectrometer. Although the device oscillates in the continuous-wave mode, measurements were made in the pulsed mode with a lock-in technique in order to eliminate surrounding noise. The pulse width and repetition rate are $0.3 \mathrm{~ms}$ and $300 \mathrm{~Hz}$, respectively. The change of characteristics with the pulse width was negligibly small. As shown in Fig. 5 (c), fundamental oscillations at 737 and $831 \mathrm{GHz}$ were obtained in forward and reverse bias conditions, respectively. The frequency was higher for the reverse bias due to the thicker spacer layer.

\section{High frequency oscillation}

Figure 6 shows the oscillation frequency as a function of RTD mesa area. The frequency increases with decreasing mesa area. However, there exists a limit of the area above which NDC cannot compensate the loss of the slot antenna.

Theoretical curves calculated with the model described in [10] are also shown in Fig. 6. The electron velocity in the collector region is an important parameter, because NDC decreases with frequency due to the transit 
time determined by the velocity. If we assume that the velocity equals to the peak velocity reported for GaInAs, the theory predicts a much smaller RTD area for the oscillation limit and higher frequency, compared with the measurement. The measurement agrees with the theoretical curve assuming a velocity smaller than the peak velocity. One possibility of the reason for the low velocity is the electron transition from the $\Gamma$ band to $L$ band due to high electric field.

Assuming that the $\Gamma$-L transition is the dominant effect, we introduced structures shown in Fig. 7 to suppress this transition and to further increase the oscillation frequency. Fig. 7 (a) shows the spike-doped structure in which the built-in potential reduces the electric field in the collector region. By this structure, the oscillation frequency was able to be increased up to $898 \mathrm{GHz}$ [40]. However, further increase was difficult because of the carrier generation from the doping which resulted in a large capacitance.

Figure 7 (b) shows the structure with graded emitter [13]. By this structure, the electric field in the collector region is reduced, because the conduction band edge of the emitter aligned to the quantum level in the well at low bias voltage. Fig. 8 (a) shows measured $I-V$ characteristics of the structures with and without the graded emitter. The peak voltage was reduced with the graded emitter, as shown in the figure. However, the peak current density decreased at the same time because the top of the tunneling barriers remained at high level due to low electric field. By introducing thin barriers, the peak current density recovered and even increased, as shown in Fig. 8 (a). The graded emitter and thin barriers are expected to reduce the collector transit time and tunneling time, respectively.

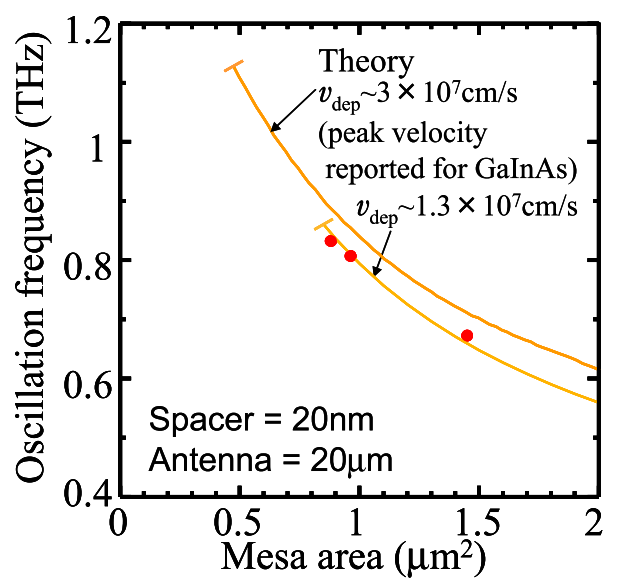

Fig. 6. Oscillation frequency as a function of mesa area of RTD with thick spacer layer.

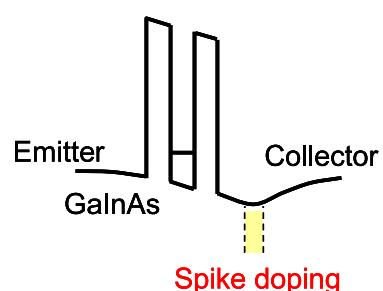

(a)

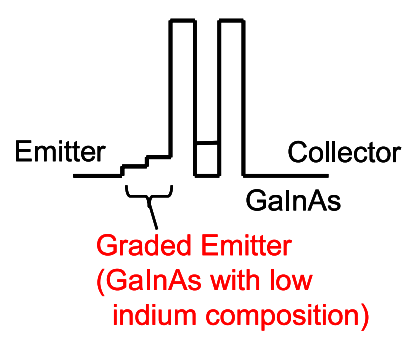

(b)

Fig. 7. Structures for the suppression of electron transition between $\Gamma$ and $\mathrm{L}$ bands. (a) Spike doping and (b) graded emitter. 
Figure 8 (b) shows the oscillation spectrum of the RTD with the graded emitter and thin barriers [13]. A fundamental oscillation at $1.04 \mathrm{THz}$ was obtained at room temperature. To our knowledge, this is the first achievement of oscillation above $1 \mathrm{THz}$ in a room-temperature electronic single oscillator. The measured output power is shown as a function of oscillation frequency in Fig. 9.

From the comparison between the above measurement and theory, the tunneling and transit times were shown to be reduced in the structure with graded emitter and thin barriers [13]. However, the reduction in the transit time was not sufficient, because the electron energy was still close to the $\mathrm{L}$ band edge. The use of materials with low effective mass and large $\Gamma-\mathrm{L}$

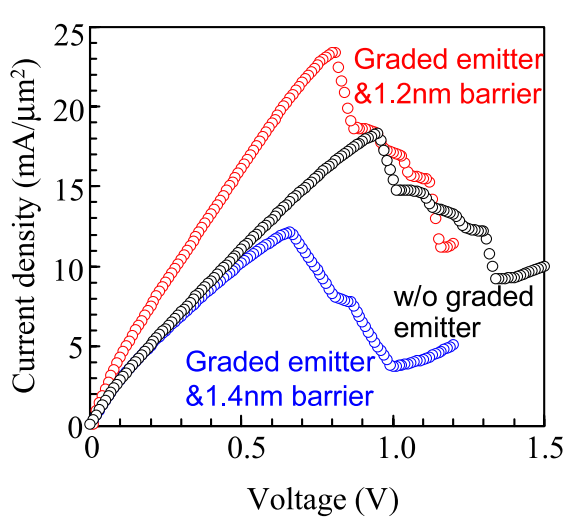

(a)

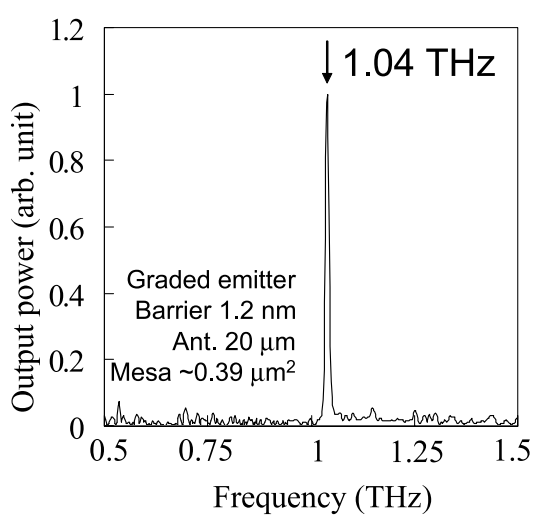

(b)

Fig. 8. Measured characteristics of RTD oscillator with graded emitter and thin barriers. (a) current voltage curves and (b) oscillation spectrum.

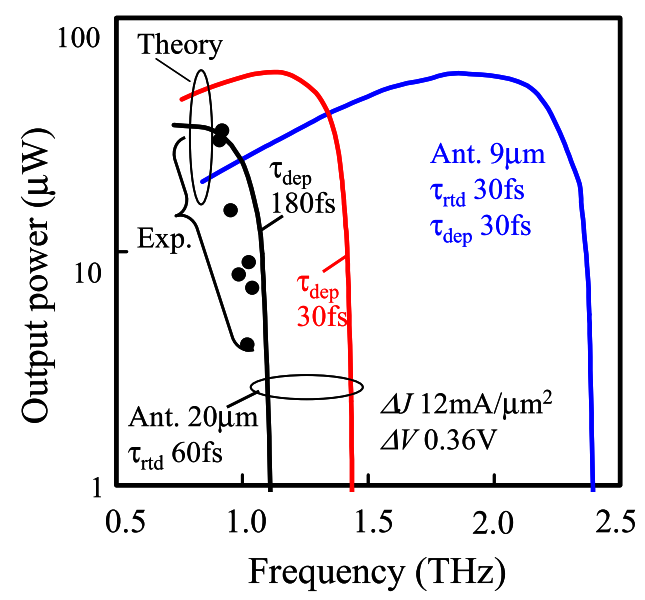

Fig. 9. Output power as a function of frequency for RTD oscillators with various tunneling and transit times $\left(\tau_{\text {rtd }}\right.$ and $\left.\tau_{\text {dep }}\right)$. Black line: the structure with graded emitter and thin barriers in Fig. 8 (a), red line: without $\Gamma$-L transition, and blue line: without $\Gamma$-L transition and with reduced tunneling time. 
separation may be effective for high electron velocity. As a preliminary experiment, an oscillation at $1.08 \mathrm{THz}$ has been obtained with the insertion of high-indium-composition GaInAs layers into the collector region right next to the exit of the barrier [41].

Theoretical calculations are shown in Fig. 9 for output power as a function of frequency under the assumptions that the $\Gamma$ - $\mathrm{L}$ transition is eliminated and that the tunneling time is reduced. The $\Gamma$ - $\mathrm{L}$ transition can be eliminated by the potential profile in which the $\Gamma$ and $\mathrm{L}$ band edges are well separated and flat over the collector region under an applied electric field. This design is possible with an appropriate heterostructures. The tunneling time can also be reduced by the adjustment of the level in the well. With these optimizations, oscillations above $2 \mathrm{THz}$ are theoretically possible, as shown in Fig. 9.

\section{Structures for high output power oscillation}

\subsection{Offset structure}

The output power of RTD oscillators is usually small ( $\sim 10 \mu \mathrm{W}$ or less). Theoretically, the maximum output power that can be extracted from the RTD is $(3 / 16) \Delta I \Delta V[10,42]$, where $\Delta I$ and $\Delta V$ are the widths of NDC region, as shown in Fig. 2 (b), which can reach $1 \mathrm{~mW}$ for the RTDs discussed here. However, the radiation conductance of the antenna is too small at present to satisfy the condition for the maximum output power given by radiation conductance $=|\mathrm{NDC}| / 2$ neglecting the antenna loss. Although the radiation conductance has a peak at some resonance frequencies of the antenna, the actual oscillation frequency is far below these frequencies due to the capacitance of RTD, resulting in the small radiation conductance.

To solve this problem, we proposed the offset slot antenna, as shown in Fig. 10 (a) $[43,44]$, in which the position of the RTD is shifted from the center of the slot. In this structure, the oscillation frequency and radiation conductance are almost independently determined by the lengths of the short and long parts of the slot, respectively. The radiation conductance can be adjusted to its peak with the oscillation frequency fixed.

Fig. 10 (b) shows theoretical calculations of output power and oscillation frequency as a function of the offset $\delta$ defined by $\delta=s /(l / 2)$, where $s$ is the displacement of RTD from the center, and $l$ is the antenna length $[43,44]$. The output power increases with increasing offset and reaches the maximum corresponding to the peak of radiation conductance. The oscillation frequency increases rapidly around the maximum output power. The output powers of about $800 \mu \mathrm{W}$ at $400 \mathrm{GHz}$ and $300 \mu \mathrm{W}$ at $600 \mathrm{GHz}$ are expected in this calculation. A higher output power is possible in RTDs with higher peak current densities. In the experiment, $420 \mu \mathrm{W}$ at $550 \mathrm{GHz}$ has been obtained up to now in RTDs with thin barriers and high peak current density shown in Fig. 8 (a) [35].

The radiation conductance can be adjusted to its peak by the offset structure and approaches the condition of the maximum output power mentioned above. However, further improvement of the antenna structure is still neces- 

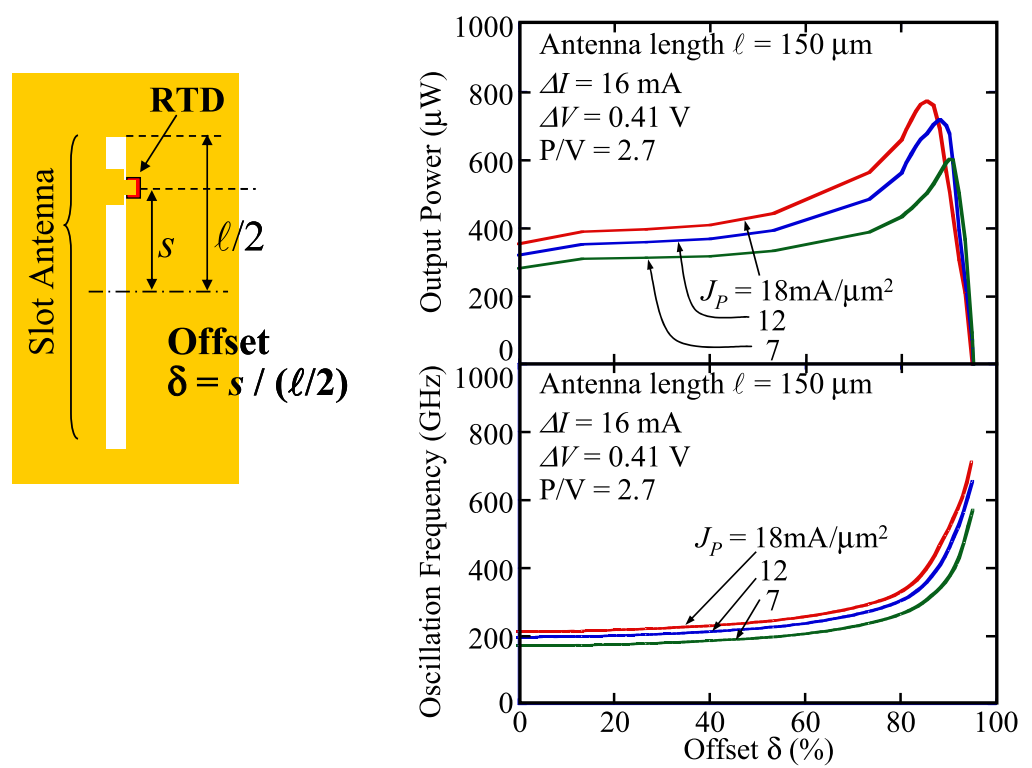

Fig. 10. Output power and oscillation frequency as a function of offset d defined as shown in Fig. 11.

sary to completely satisfy this condition. This is in progress now. The loss of the antenna other than the radiation must also be suppressed.

\subsection{Array configuration}

The power combining using an array configuration is an effective method for high output power $[45,46,47,48,49,50,51]$. To obtain a combined high output power in the array configuration, all of the array elements are required to coherently oscillate with the same frequency. This condition can be satisfied by utilizing mutual injection locking between the elements. We reported observation of the mutual injection locking $[45,46]$ and theoretical analysis of the coupling condition between the array elements required for stable locking [52].

Figure 11 shows an experimental result of three-element array in which the oscillator elements are coupled with each other through angled slot lines [53]. A single peak was observed in the spectrum due to mutual injection locking. Although the output power of each element is as small as $2-3 \mu \mathrm{W}$ due to small current density in this experiment, the combined output power observed in the front direction was larger than the simple sum of the output power of each element.

Theoretically, the output electric fields from all the elements are coherently combined, and the whole device works as a coherent antenna array. Thus, the output power concentrates into the front direction through the improvement of the antenna directivity. The radiation conductance also increases slightly. Due to these effects, the output power in the front direction is nearly proportional to the square of the element number, if the free-running frequencies of the elements are equal with each other [52]. Although the output power decreases with the divergence in the free-running frequencies before the locking [52], the above effects are retained. The measured output 
power in Fig. 11 may indicate this situation.

Applying the array configuration to the high power oscillators with offset slot antennas and high current density RTDs described above, output power of more than $1 \mathrm{~mW}$ will be possible at $1 \mathrm{THz}$ with more than about 5 elements.

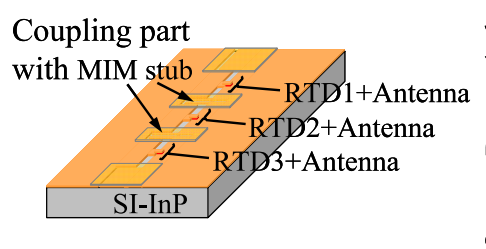

(a)

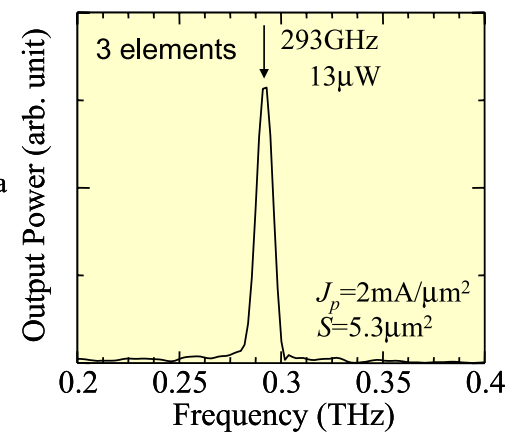

(b)

Fig. 11. Array configuration of RTD oscillators. (a) Structure of planar-coupled three -element array and (b) oscillation spectrum.

\section{Spectral characteristics and direct modulation}

Spectral characteristics are important for various applications of $\mathrm{THz}$ sources. We investigated spectral characteristics of RTD oscillators using heterodyne detection system shown in Fig. 12 as a preliminary experiment [54].

The output of RTD (left-hand side of Fig. 12) is extracted through a Si hemispherical lens and mixed with the output of the local oscillator (LO) with a Si plate, and detected with the Schottky-barrier diode (SBD, righthand side). The oscillation frequency of the RTD is $550 \mathrm{GHz}$. The LO is composed of a microwave signal generator and multipliers, and its output frequency is $279 \mathrm{GHz}$. The second harmonic heterodyne was employed. The fundamental heterodyne was also reported recently with an RTD as LO [55].

For the detector, we fabricated an InP SBD with a broad-band bow-tie antenna, as shown in the right-hand side of Fig. 12. InP SBD has the advantage of possible integration with InP-based high-speed devices, which may be useful for signal processing, in particular in high-speed wireless communication systems.

Figure 13 shows the heterodyne-detected signal [54]. Although the measured spectrum was slowly fluctuated probably due to external noise, the instantaneous linewidth was obtained from Fig. 13 (a) to be less than 10 MHz. The theoretical linewidth was about $6 \mathrm{MHz}[54,56]$, which was consistent with the measurement. The linewidth is inversely proportional to square of the amplitude of oscillation voltage across the RTD. A narrow linewidth may be possible by improvement of RTD and antenna structures to obtain the large amplitude. The phase-locked loop (PLL) system making use of the 


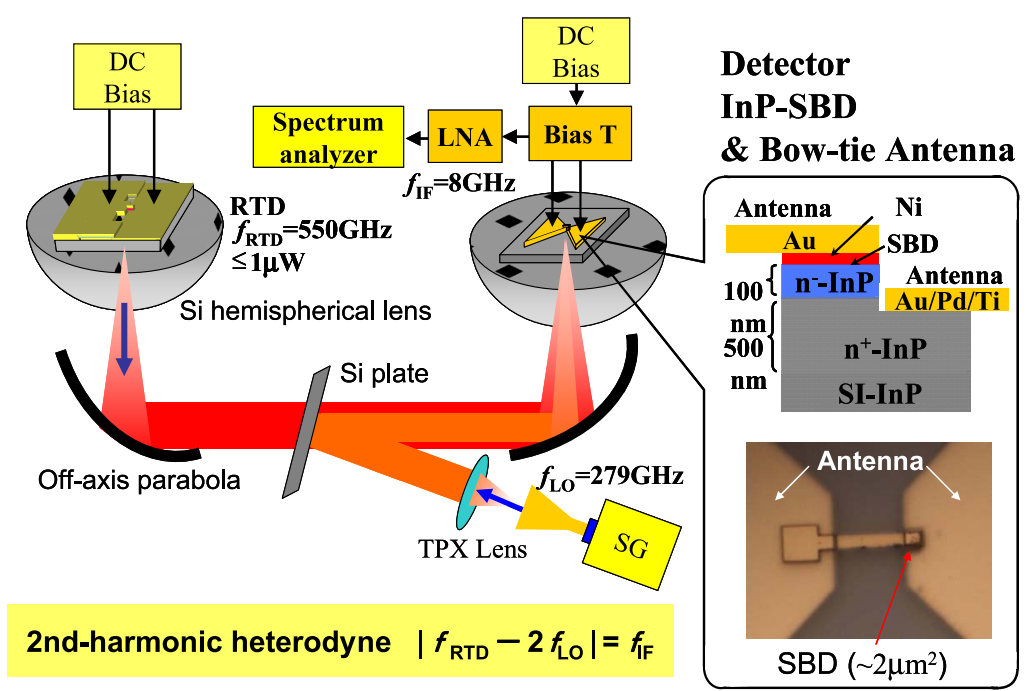

Fig. 12. Measurement setup of heterodyne detection of output from RTD oscillator.

voltage controlled oscillation (VCO) mentioned below is also effective for a stable and narrow linewidth.

Frequency change with bias voltage, i. e., the VCO, was observed, as shown in Fig. 13 (b). The total change of the frequency was typically 1$5 \%$. The mechanism of this change is the bias-dependent tunneling time, which produces the bias-dependent additional capacitance and the frequency change $[57,58,59,60,61]$. This frequency change was also observed in the RTD oscillating above $1 \mathrm{THz}$ shown in Fig. 8 [13]. This property is useful for precise control and stabilization of frequency with the PLL system.

The direct modulation of RTD oscillators with bias voltage is useful for the application to simple wireless communication systems [62]. Whether the modulation is that in intensity or frequency depends on bias point, because the frequency change is large around the bias point at the peak output power and saturates in the bias region above this point $[13,60]$. The modulation frequency is limited at present by the large capacitance of the overlapped metal-insulator-metal (MIM) layers at the edges of the slot antenna shown in Fig. 4. By reducing the area of the MIM layers, the $3 \mathrm{~dB}$ cut-off frequency of about $3.2 \mathrm{GHz}$ was obtained in a preliminary measurement of the intensity modulation [63]. Higher frequency is possible by further reducing the area of MIM layers and also by removing parasitic elements. Filtering circuits instead of the MIM layers which reflect the THz frequency may also be effective for high frequency modulation.

\section{Conclusion}

Recent results of THz oscillators with RTDs are described. By the structure reducing the transit time, the frequency further increased to $1.04 \mathrm{THz}$. This is the first achievement of a fundamental oscillation above $1 \mathrm{THz}$ in roomtemperature electronic single oscillators. The output power of $400 \mu \mathrm{W}$ at $550 \mathrm{GHz}$ was obtained in a single oscillator by the offset-fed slot antenna. Co- 


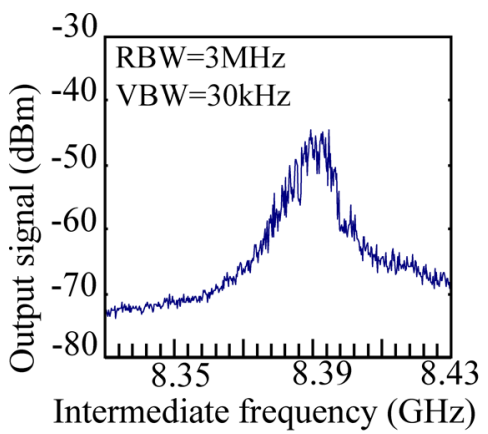

(a)

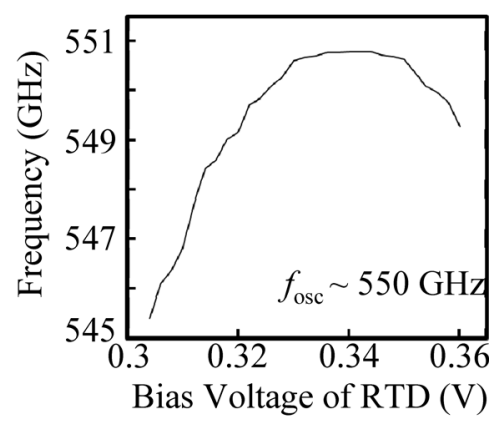

(b)

Fig. 13. Heterodyne-detected signal of output from RTD oscillator. (a) Spectrum and (b) frequency variation with bias voltage.

herent power combining with multi-element array was observed. The spectral linewidth, frequency change with bias voltage, and direct modulation were also described.

Based on these results, we believe that RTD oscillator is a possible candidate for compact and coherent THz sources. Making use of the advantage of room temperature oscillation and integralibility with other high-speed electron/optical devices, wide applications may be expected, in particular in short-distance high-speed simple wireless communication systems.

\section{Acknowledgments}

The authors thank Emeritus Professors Y. Suematsu and K. Furuya of the Tokyo Institute of Technology for continuous encouragement. The authors also thank Professor S. Arai, Associate Professors Y. Miyamoto, M. Watanabe, and N. Nishiyama of the Tokyo Institute of Technology, Doctors I. Hosako and N. Sekine of the National Institute of Communications Technology, Doctors T. Enoki, M. Ida, N. Shigekawa, S. Yamahata, H. Sugiyama, and H. Yokoyama of NTT Corporation for stimulating and fruitful discussions. This work was supported by a grant-in-aid from the Ministry of Education, Culture, Sports, Science and Technology.

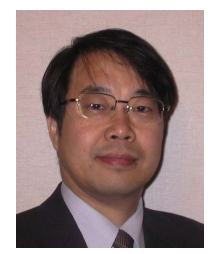

\footnotetext{
Masahiro Asada

received the B.E., M.E., and Dr. Eng. degrees in Physical Electronics from the Tokyo Institute of Technology, in 1979, 1981, and 1984, respectively. In 1984, he joined the Department of Physical Electronics, Tokyo Institute of Technology, as a research associate. From 1986-1987, he was with the Physics Institute of Stuttgart University, Stuttgart, Germany, as a Research Fellow of the Alexander von Humboldt Foundation. From 1988-1999, he was an associate professor in the Department of Electrical and Electronic Engineering, Tokyo Institute of Technology. Since 1999 he has been a professor in the Interdisciplinary Graduate School of Science and Engineering, Tokyo Institute of Technology. Currently, he is interested in high-frequency electron devices, especially terahertz devices using nanostructures. Dr. Asada is a Fellow of the Japan Society of Applied Physics and a senior member of the IEEE.
} 


\section{Safumi Suzuki}

received the B.E. degree in Electrical and Electronic Engineering, and the M.E. and D.E. degrees in Electronics and Applied Physics from the Tokyo Institute of Technology, in 2005, 2007, and 2009, respectively. Since 2009, he has been an assistant professor in the Interdisciplinary Graduate School of Science and Engineering, Tokyo Institute of Technology. He is presently engaged in research on terahertz electron devices. Dr. Suzuki is a member of the Japan Society of Applied Physics. 